\title{
Sequential Responses of Adenocarcinoma of the Lung to Erlotinib after Gefitinib in Never Smoker Korean Woman
}

\author{
Hoon-Kyo Kim, M.D. ${ }^{1}$, Myeong Im Ahn, M.D. ${ }^{2}$, Jinyoung Yoo, M.D. ${ }^{3}$, Chi Hong Kim, M.D. ${ }^{1}$, Hong-Jun \\ Yang, M.D. ${ }^{1}$ and Byoung Yong Shim, M.D. ${ }^{1}$ \\ Lung Cancer Center; Departments of ${ }^{1}$ Internal Medicine, ${ }^{2}$ Diagnostic Radiology and ${ }^{3}$ Pathology, St. Vincent's \\ Hospital, The Catholic University of Korea, Suwon, Korea
}

A patient with adenocarcinoma of the lung was treated sequentially using two kinds of EGFR tyrosine kinase inhibitors, gefitinib and erlotinib. The patient was a 73-year-old female who received gefitinib as a second line treatment, which resulted in a partial response with response duration of 6 months. After progression of the disease, the patient received erlotinib, which resulted in partial response again with

\section{CASE REPORT}

A 73-year-old woman presented in the clinic with multiple lung nodules. Neither she nor her husband had ever smoked. An initial chest x-ray showed a main lung mass in the right middle lobe with variable sized nodules in both lungs (Fig. 1). Bronchoscopy did not show a definite endobronchial lesion. A CT-guided needle biopsy of the right middle lobe mass showed moderately differentiated adenocarcinoma. The patient was treated with 4 cycles of gemcitabine and cisplatin between September and December 2003; the disease remained stable after 2 cycles of chemotherapy.

A chest x-ray taken on February 2004 showed an increased size and number of nodules in both lungs (Fig. 2). However, the patient remained free of any respiratory or constitutional symptoms. On March 2004, the patient was started on gefitinib, $250 \mathrm{mg}$ a day, and continued this treatment until September 2004 for 6 months. Gefitinib treatment resulted in a remarkable response with a decrease in the size and number of bilateral lung nodules (Fig. 3). The patient tolerated the treatment with only grade 1 skin toxicity. After 6 months of treatment, gefitinib was discontinued due to disease progression.

The patient refused further therapy despite of progression of the lung lesions (Fig. 4). On April 2005, when erlotinib became available in Korea, the patient was started on erlotinib, $150 \mathrm{mg}$ a day. There was a remarkable tumor res-

Correspondence: Chi Hong Kim, Department of Internal Medicine, St. Vincent's Hospital, The Catholic University of Korea, 93, Ji-dong, Paldal-gu, Suwon 442-723, Korea. (Tel) 82-31-249-7125, (Fax)

82-31-253-8898, (E-mail) chihongk@yahoo.co.kr

Received January 5, 2007, Accepted January 27, 2007 response duration of $\mathbf{1 1 . 5}$ months. This observation suggests that treatment with erlotinib may be effective in patients who develop progressive disease after a primary treatment with gefitinib following an initial response. (Cancer Res Treat. 2007;39:37-39)

Key Words: Lung neoplasm, Targeted therapy, Erlotinib, Gefitinib

ponse that qualified for partial response after erlotinib therapy again (Fig. 5).

Grade 1 skin toxicity developed after the administration of erlotinib. The patient was able to take erlotinib for 11.5 months until disease progression was again noted.

Eventually, the patient developed multiple brain metastases on May 2006 and died of lung cancer with accompanying brain metastases. She lived for 33 months after the diagnosis of stage IV adenocarcinoma of the lung and for 26 months after the administration of gefitinib.

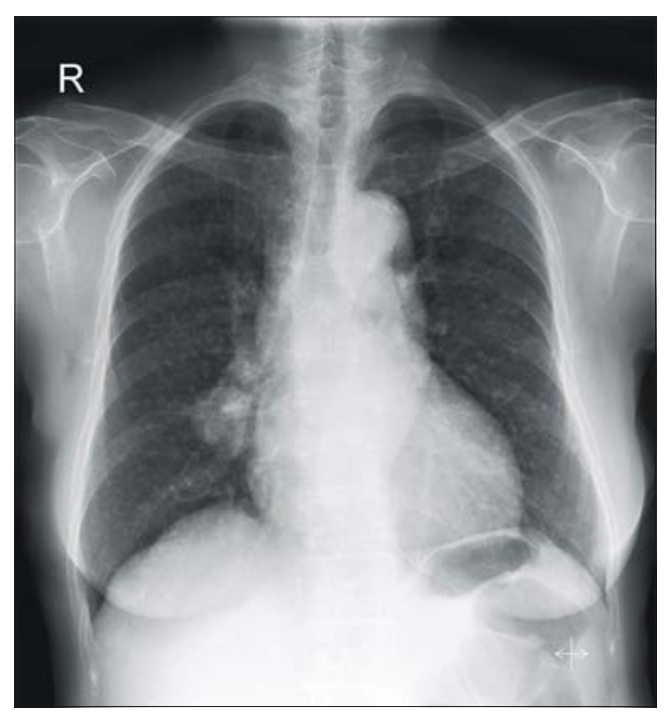

Fig. 1. Initial chest PA (September, 2003) shows a $3.7 \mathrm{~cm}$ sized lobulated mass in the right infrahilar region, with innumerable small round nodules in both lungs. 


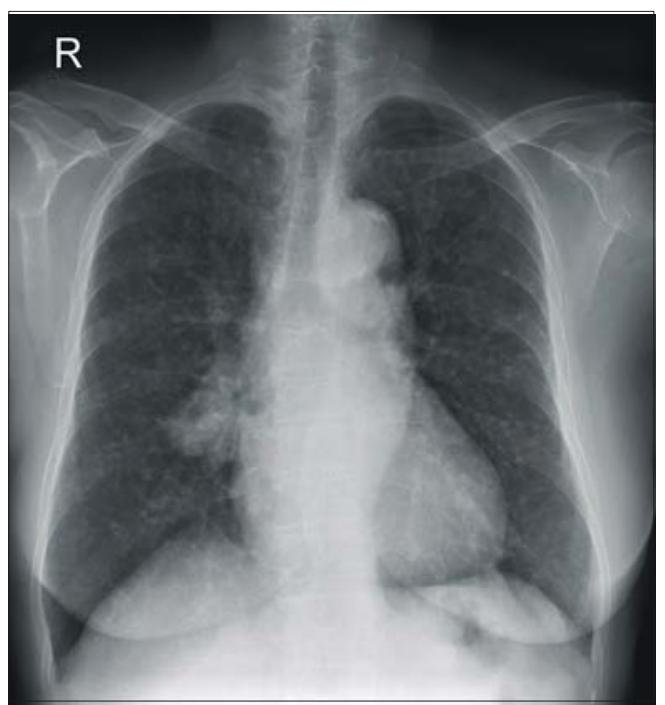

Fig. 2. Follow-up chest PA (February, 2004) shows slightly increased size of the primary lung ca. and metastatic nodules in both lungs.

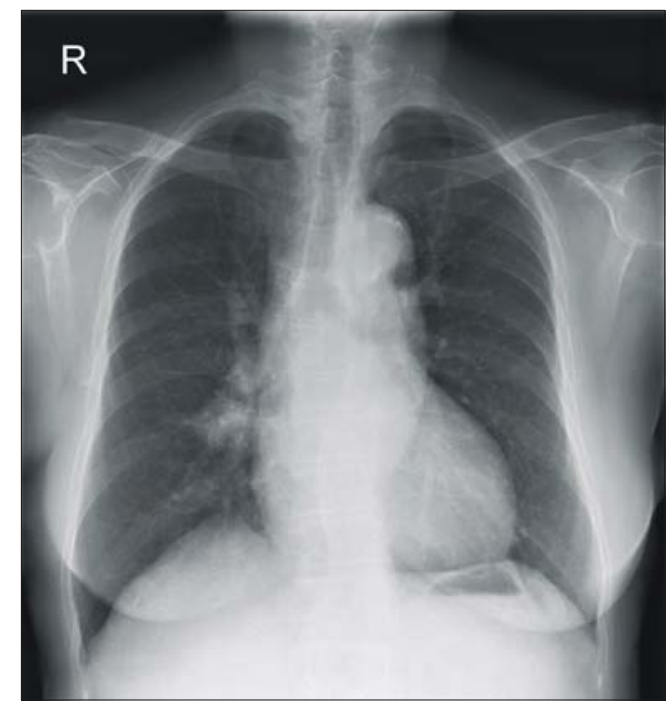

Fig. 3. Chest PA during Getifinib therapy (May, 2004) shows decreased size of the primary lung ca. and metastatic lung nodules.

\section{DISCUSSION}

On March 2005, the U.S. Food and Drug Administration (FDA) withdrew the approval of gefitinib based on the results of the ISEL Study (1) that compared the use of gefitinib with placebo. While the ISEL study failed to demonstrate any of survival advantages of using gefitinib over placebo, high response rates were observed in a sugbgroup of patients: those who never-smoked, women, those with adenocarcinoma and those of Asian origin. A survival benefit was observed in

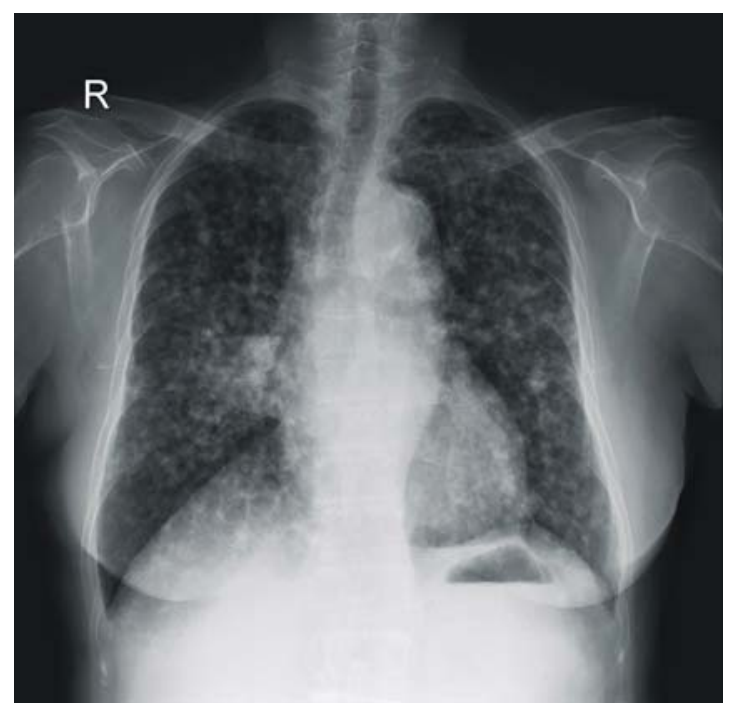

Fig. 4. Chest PA at the start of Erlotinib treatment (April, 2005) shows marked aggravation of metastatic nodules in both lungs, obscuring the right middle lobe lung ca..

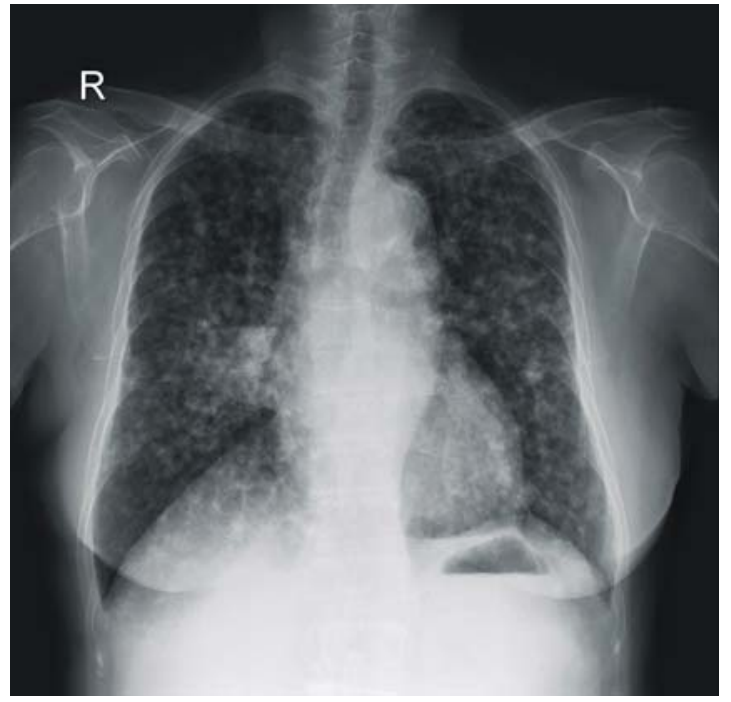

Fig. 5. Chest PA during Erlotinib treatment (October, 2005) represents markedly regressed diffuse metastatic nodules in both lungs. Right pleural effusion appears to be unchanged.

patients who were never smokers and those who are of Asian origin (1). In another study conducted in Korea, patients with an EGFR mutation showed a higher response rate to gefitinib treatment with a significant survival benefit (2). Although gefitinib was withdrawn in the US market and is rarely used in Europe, gefitinib is still used in many of Asian countries including Korea, Taiwan, China and Japan. Its continued use is justified based on data of a subset analysis of the ISEL study and other studies which showed not only a high response rate but also a survival benefit in Asian women who never smoked and who demonstrate adenocarcinoma pathology (3). 
A similar, but not identical EGFR tyrosine kinase inhibitor, erlotinib was approved November 2004 by the US. FDA for use in patients who had failed on a previous chemotherapy regimen. A randomized, placebo-controlled Phase III trial (BR.21) (4) demonstrated a survival advantage for treatment with erlotinib in all patient subsets with the greatest benefit shown in never-smokers with adenocarcinoma and those of Asian origin. In multiple logistic-regression analyses, neversmokers, the presence of adenocarcinoma, and EGFR expression were associated with erlotinib responsiveness (4). However, to our best knowledge, there have been few case reports describing the outcome of sequential treatment with two different EGFR tyrosine kinase inhibitors.

Our case may be the first ever reported in Korea that responded sequentially to erlotinib therapy following initial therapy with gefitinib.

Viswanathan and colleagues (5) reported a lack of response to erlotinib after progression on gefitinib therapy in 4 women who responded to gefitinib initially and in 1 man who was refractory to treatment with gefitinib. However, Garfield (6) reported a response to treatment with erlotinib in a gefitinib refractory man. Choong and colleagues (7) reported a response to gefitinib therapy in an erlotinib refractory Japanese-American woman who had never smoked, and who had a novel double L858R+E884K somatic mutation in the EGFR gene. An explanation for a response in this patient was not given.

Our experience suggests that erlotinib therapy could be administered to patients who develop progressive disease after initial therapy with gefitinib that has produced an initial response.

\section{REFERENCES}

1. Thatcher N, Chang A, Parikh P, Pereira JR, Ciuleanu T, von Pawel J, et al. Gefitinib plus best supportive care in previously treated patients with refractory advanced non-small-cell lung cancer:results from a randomized, placebo-controlled, multicentre study (Iressa Survival Evaluation in Lung Cancer). Lancet. 2005;366:1527-37.

2. Han SW, Kim TY, Hwang PG, Jeong S, Kim J, Choi IS, et al. Predictive and prognostic impact of epidermal growth factor receptor mutation in non-small-cell lung cancer patients treated with gefitinib. J Clin Oncol. 2005;23:2493-501.

3. Lee DH, Han JY, Lee HG, Lee JJ, Lee EK, Kim HY, et al. Gefitinib as a first-line therapy of advanced or metastatic adenocarcinoma of the lung in never-smokers. Clin Cancer Res. 2005;11:3032-7.

4. Shepherd FA, Pereira JR, Ciuleanu T, Tan EH, Hirsh V, Thongprasert $\mathrm{S}$, et al. Erlotinib in previously treated nonsmall-cell lung cancer. N Engl J Med. 2005;353:123-32.

5. Viswanathan A, Pillot G, Govindan R. Lack of response to erlotinib after progression on gefitinib in patients with advanced non-small cell lung cancer. Lung Cancer. 2005;50:417-8.

6. Garfield DH. Response to Erlotinib after failure of Gefitinib in a patient with advanced non-small-cell lung carcinoma. J Clin Oncol. 2005;23:7738-40.

7. Choong NW, Dietrich S, Seiweert TY, Tretiakova MS, Nallasura V, Davies GC, et al. Gefitinib response of erlotinibrefractory lung cancer involving meninges-role of EGFR mutation. Nat Clin Pract Oncol. 2006;3:50-7. 